\title{
Single-Dose Oritavancin Treatment of Acute Bacterial Skin and Skin Structure Infections: SOLO Trial Efficacy by Eron Severity and Management Setting
}

\author{
Daniel H. Deck · Jennifer M. Jordan · Thomas L. Holland • \\ Weihong Fan · Matthew A. Wikler · Katherine A. Sulham · \\ G. Ralph Corey
}

Received: May 16, 2016/ Published online: July 1, 2016

(c) The Author(s) 2016. This article is published with open access at Springerlink.com

\section{ABSTRACT}

Introduction: Introduction of new antibiotics enabling single-dose administration, such as oritavancin may significantly impact site of care decisions for patients with acute bacterial skin and skin structure infections (ABSSSI). This analysis compared the efficacy of single-dose oritavancin with multiple-dose vancomycin in patients categorized according to disease severity via modified Eron classification and management setting.

Methods: SOLO I and II were phase 3 studies evaluating single-dose oritavancin versus 7-10 days of vancomycin for treatment of ABSSSI. Patient characteristics were collected at baseline and retrospectively analyzed. Study protocols were amended, allowing outpatient

Enhanced content To view enhanced content for this article go to www.medengine.com/Redeem/CFD4F0603 7897AC2.

D. H. Deck $(\bowtie) \cdot$ J. M. Jordan $(\bowtie) \cdot$ W. Fan •

M. A. Wikler - K. A. Sulham

The Medicines Company, Parsippany, NJ, USA

e-mail: Daniel.Deck@THEMEDCO.com

J. M. Jordan

e-mail: Jennifer.jordan@themedco.com

T. L. Holland · G. Ralph Corey

Duke University Hospital, Durham, NC, USA management at the discretion of investigators. In this post hoc analysis, patients were categorized according to a modified Eron severity classification and management setting (outpatient vs. inpatient) and the efficacy compared.

Results: Overall, 1910 patients in the SOLO trials were categorized into Class I (520, 26.5\%), II $(790,40.3 \%)$, and III $(600,30.6 \%)$. Of the 767 patients $(40 \%)$ in the SOLO trials who were managed entirely in the outpatient setting $40.3 \%$ were categorized as Class II and 30.6\% were Class III. Clinical efficacy was similar between oritavancin and vancomycin treatment groups, regardless of severity classification and across inpatient and outpatient settings. Class III patients had lower response rates (oritavancin 73.3\%, vancomycin $76.6 \%$ ) at early clinical evaluation when compared to patients in Class I (82.6\%) or II (86.1\%); however, clinical cure rates at the post-therapy evaluation were similar for Class III patients (oritavancin 79.8\%, vancomycin 79.9\%) when compared to Class I and II patients (79.1-85.7\%).

Conclusion: Single-dose oritavancin therapy results in efficacy comparable to multiple-dose 
vancomycin in patients categorized according to modified Eron disease severity classification regardless of whether management occurred in the inpatient or outpatient setting.

Funding: The Medicines Company, Parsippany, NJ, USA.

Trial registration: ClinicalTrials.gov identifiers, NCT01252719 (SOLO I) and NCT01252732 (SOLO II).

Keywords: Acute bacterial skin and skin structure infections (ABSSSI); Eron classification; Oritavancin; Outpatient

\section{INTRODUCTION}

Oritavancin is a lipoglycopeptide antibiotic approved by the Food and Drug Administration (FDA) and the European Medicines Agency (EMA) for use as a single-dose treatment of acute bacterial skin and skin structure infections (ABSSSI). Approvals were based on results from two identical Phase 3 clinical trials (SOLO I and II; ClinicalTrials.gov identifiers, NCT01252719 and NCT01252732, respectively) of a single intravenous (IV) dose of oritavancin compared to multiple-dose vancomycin administered IV for 7-10 days $[1,2]$. Both clinical trials demonstrated that a single-dose of oritavancin was non-inferior to multiple-day vancomycin therapy. Since a full course of therapy is delivered in a single IV dose, oritavancin has the potential to shift the treatment of ABSSSI to the outpatient setting without compromising efficacy and without the need for laboratory monitoring (as is required with vancomycin) or an indwelling IV catheter [3]. This approach can affect how ABSSSI is managed, by reducing or in some cases eliminating costs and risks of hospitalization.
Shifting the care of ABSSSI to the outpatient setting requires appropriate patient selection based on severity of illness and patient-specific comorbidities that may impact treatment outcomes or require a higher level of care. Evidence-based treatment guidelines or pathways of care are increasingly used to select the most appropriate treatment, including site of care decisions. Although skin and skin structure infections are extremely common, there is a lack of validated evidence-based schemes for the classification of clinical presentation, disease severity, impact of comorbidities, and site of care. Several classification systems and treatment algorithms have been published in recent years in attempts to identify which patients should be treated as inpatients versus outpatients, the route of antibiotic administration, and antibiotic choice [4-9]. The Eron classification was developed by an expert panel of clinicians and researchers to categorize severity to guide initial site of care decisions for patients with skin and soft tissue infections [5]. In the Eron classification, patients are grouped into four categories of ascending severity according to signs and symptoms of infection and comorbidities. Class I patients have no signs or symptoms of systemic toxicity, have no uncontrolled comorbidities that may complicate treatment and usually can be managed on an outpatient basis. Class II patients are either systemically ill, but any comorbidities they may have are stable, or are systemically well but have one or more comorbidities. Class III patients may appear toxic, or they may appear nontoxic but have unstable comorbidities that may interfere with their response to therapy. Class III patients usually require initial inpatient treatment but many can be quickly discharged on outpatient 
parenteral antimicrobial therapy (OPAT) or oral therapy. Class IV patients have sepsis syndrome or serious life-threatening infections (e.g., necrotizing fasciitis) and should be admitted for stabilization. The Eron classification has since been adapted by the Clinical Resource Efficiency Support Team (CREST) into the 'Guidelines on the Management of Cellulitis in Adults' (CREST guidelines) which are used widely in the UK [6].

In view of the potential for outpatient management of a substantial number of patients, a clinical algorithm derived from the Eron classification was used for a post hoc analysis to evaluate patients enrolled in the SOLO trials. Clinical efficacy of the single-dose of oritavancin was compared to multiple-dose vancomycin based on the modified Eron classification and setting of care.

\section{METHODS}

SOLO I and II were two identical, phase 3, multi-center, randomized, double-blind studies that compared the efficacy and safety of a single $1200 \mathrm{mg}$ dose of IV oritavancin to vancomycin $1 \mathrm{~g}$ IV twice daily for 7-10 days in adults with ABSSSI [1, 2]. Patients randomized to oritavancin received placebo infusions twice daily to maintain treatment blinding. The SOLO I and SOLO II protocols were amended during the trials to allow patients to be managed in the outpatient setting at the discretion of the investigator. The SOLO trials study design was consistent with current regulatory guidelines for eligibility criteria, end points, assessment methods and non-inferiority margins.

Eligible patients were at least 18 years of age and had received a diagnosis of ABSSSI that was suspected or proven to be caused by a
Gram-positive pathogen and that required at least 7 days of IV therapy. The diagnosis of ABSSSI required the presence of wound infection (either traumatic or surgical in origin), cellulitis, or a major cutaneous abscess, with each lesion surrounded by erythema, edema, or an area of induration of at least $75 \mathrm{~cm}^{2}$. Signs and symptoms of systemic inflammation were also required. Patients were not eligible to participate if they received systemic or topical antibacterial therapy with Gram-positive activity within the preceding 14 days unless the documented failure to previous therapy was available. Patients were excluded if they had suspected or confirmed bacteremia, severe sepsis or refractory shock, or any evolving, necrotizing infection (i.e., necrotizing fasciitis).

Clinical evaluations were performed at: (1) early clinical evaluation (ECE) 48-72 $\mathrm{h}$ after the initiation of the therapy; (2) the end of therapy (EOT) from Day 7 to Day 10; (3) Day 10 evaluation; (4) post-therapy evaluation (PTE) in 7-14 days after the EOT; (5) safety follow-up at 60 days (+7 days; Fig. 1). The primary efficacy endpoint was a composite outcome at ECE comprised (1) cessation of spreading or reduction in the size of the baseline lesion, (2) absence of fever, and (3) no rescue antibiotic medication. The key secondary endpoint was investigator-assessed clinical cure at PTE. The additional main secondary efficacy outcome was lesion size decrease by $\geq 20 \%$ from baseline at ECE.

An algorithm based on the Eron classification was developed and applied to the modified intent-to-treat (mITT) pool of SOLO patients to classify each patient enrolled in the pooled dataset from the SOLO trials. The algorithm incorporated signs and symptoms of systemic illness in addition to concomitant 


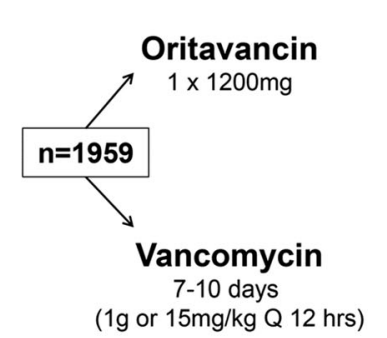

Legend:

IV Active drug

IV Placebo

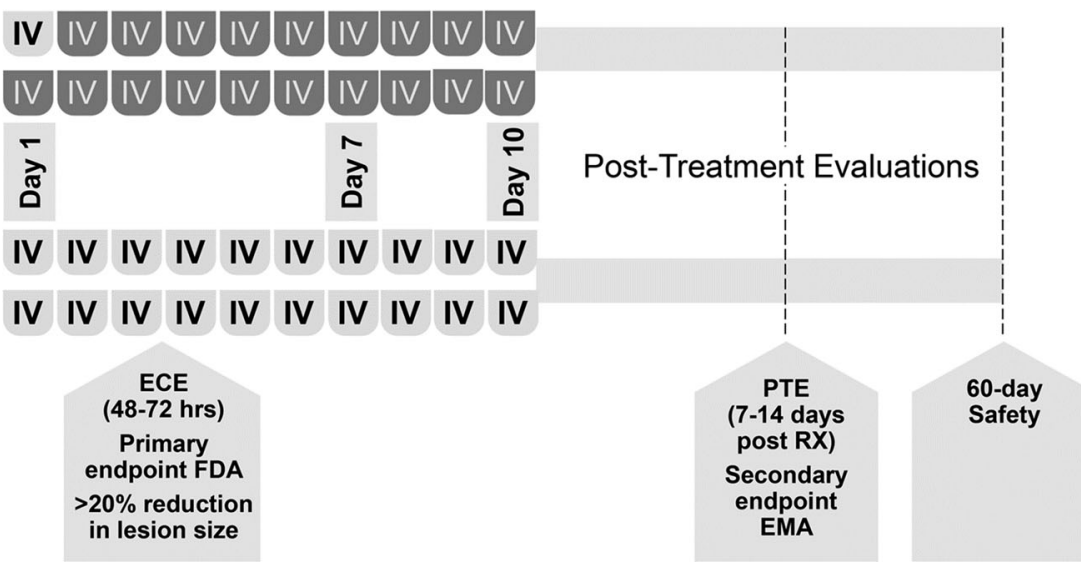

Fig. 1 SOLO study design $(n=1959$, mITT population). $E C E$ early clinical evaluation $(48-72 \mathrm{~h}$ from treatment initiation), EMA European Medicines Agency, FDA Food

medical conditions which have been implicated in poor therapeutic response (advanced age, chronic liver or renal disease, diabetes, obesity, chronic venous insufficiency) that were recorded in the case reports for each patient $[6,10]$. Clinical variables included data collected in the SOLO trials, inclusion/ exclusion criteria, and input from clinical experts. The clinical criteria are described in Table 1. Patients were classified into Class I-IV based on the presence of comorbidities and systemic symptoms of infection. Stratification criteria and assignment of patients to a modified Eron class were performed by expert consensus within a panel of seven advisors. Patients categorized as Class IV (bacteremia or absolute neutrophil count less than 500 detected after enrollment) were excluded from this analysis as they were not considered appropriate for outpatient management. Location of care in the outpatient or inpatient setting and all follow-up visits were documented in the case report form.

All procedures followed were in accordance with the ethical standards of the responsible committee on human experimentation and Drug Administration, hrs hours, $I V$ intravenous, mITT modified intent-to-treat, PTE post-therapy evaluation (7-14 days after the end of therapy), $Q$ every

(institutional and national) and with the Helsinki Declaration of 1964, as revised in 2013. Informed consent was obtained from all patients for being included in the SOLO trials. This article is based on previously conducted studies, and does not involve any new studies of human or animal subjects performed by any of the authors.

\section{Statistical Analysis}

Discrete variables were summarized as frequencies and percentages. Continuous variables were summarized as means with standard deviations (SD). Efficacy outcomes of oritavancin and vancomycin therapy according to modified Eron classification and receipt of treatment in the outpatient or inpatient setting were presented as percentages, differences and two-sided 95\% confidence intervals (CI) and compared using Chi-square testing between two treatment groups. The alpha level of significance was set to 0.05 . All $p$ values being presented were two-sided. This was a post hoc exploratory analysis that was not powered for statistical inference. All analyses were 
Table 1 Clinical criteria used to define modified Eron Classes I-IV

\begin{tabular}{|c|c|}
\hline Eron class & Clinical criteria \\
\hline \multirow[t]{2}{*}{ IV } & Bacteremia (positive blood culture); or \\
\hline & Absolute total neutrophils count $<500$ \\
\hline \multirow[t]{5}{*}{ III } & Meeting SIRS criteria \\
\hline & $\mathrm{CrCl}<20 \mathrm{~mL} / \mathrm{min}$ or on dialysis \\
\hline & ALT/AST $>10$-times ULN \\
\hline & Heart rate $>90 / \mathrm{min}$, breath rate $>20 / \mathrm{min}$, or systolic $\mathrm{BP}<90 \mathrm{mmHg}$; or \\
\hline & Cancer \\
\hline \multirow[t]{9}{*}{ II } & Age $\geq 75$ years old \\
\hline & Glucose $>11.1 \mathrm{mmol} / \mathrm{L}$ \\
\hline & Congestive heart failure at the randomization \\
\hline & $30 \mathrm{~mL} / \mathrm{min}<\mathrm{CrCl}<60 \mathrm{~mL} / \mathrm{min}$ \\
\hline & Hepatitis (excluding AST/ALT >10-times ULN) \\
\hline & Peripheral vascular disease \\
\hline & Diabetes mellitus \\
\hline & Fever (temperature $>38.0^{\circ} \mathrm{C}$ ); or \\
\hline & $\mathrm{BMI} \geq 30 \mathrm{~kg} / \mathrm{m}^{2}$ \\
\hline I & For the patients who didn't meet Classes II-IV \\
\hline
\end{tabular}

$A L T$ alanine transaminase, $A S T$ aspartate transaminase, $B M I$ body mass index, $B P$ blood pressure, $C r C l$ creatinine clearance, SIRS systemic inflammatory response syndrome, $U L N$ upper limit of normal

conducted using SAS version 9.2 (SAS Institute Inc., Cary, NC, USA).

\section{RESULTS}

Of the 1959 mITT patients in the SOLO studies, $520(26.5 \%), 790(40.3 \%)$, and $600(30.6 \%)$ were categorized into Class I, II, and III, respectively (Table 2). The 49 patients categorized into Class IV were excluded from this analysis since initial inpatient management would generally be considered standard of care for patients with bacteremia and/or neutropenia. A majority of the patients in the SOLO trials had significant comorbidities and/or systemic symptoms of infection as $70.9 \%$ of patients were categorized as Class II or III. Demographics and baseline characteristics were different across Class I-III (Table 3). Patients in Class I, had a lower incidence of comorbidities (diabetes, renal insufficiency or hepatic conditions), were younger, and had lower body mass index and smaller mean lesion size. Mean lesion size increased with severity classification. In comparison to Class I and II patients, a greater percentage of Class III patients were diagnosed with cellulitis. Of Class III patients, 55.5\% met systemic inflammatory response syndrome (SIRS) criteria (presence of two or more systemic signs of infection). 
Table 2 Distribution of SOLO patients in modified Eron Classes I-III (mITT population, $n=1959$ )

\begin{tabular}{lllll}
\hline & Class I & Class II & Class III & Total (Class I-III) \\
\hline SOLO mITT $(n=1959)$ & $520(26.5 \%)$ & $790(40.3 \%)$ & $600(30.6 \%)$ & 1910 \\
Inpatients, $n(\%)$ & $301(57.9 \%)$ & $431(54.6 \%)$ & $411(68.5 \%)$ & \\
ORI, $n$ & 144 & 224 & 203 & 571 \\
VAN, $n$ & 157 & 207 & 208 & 572 \\
Outpatients, $n(\%)$ & $219(42.1 \%)$ & $359(45.4 \%)$ & $189(31.5 \%)$ & $767(40.2 \%)$ \\
ORI, $n$ & 108 & 182 & 89 & 379 \\
VAN, $n$ & 11 & 177 & 100 & 388 \\
\hline
\end{tabular}

mITT modified intent-to-treat, $O R I$ oritavancin, $V A N$ vancomycin

Table 3 Demographics and baseline characteristics by classification (mITT population)

\begin{tabular}{llll}
\hline Parameter & Class I $(\boldsymbol{N}=\mathbf{5 2 0})$ & Class II $(\boldsymbol{N}=\mathbf{7 9 0})$ & Class III $(\boldsymbol{N}=\mathbf{6 0 0})$ \\
\hline Age, years (mean \pm SD) & $40.1 \pm 13.6$ & $47.8 \pm 13.2$ & $45.4 \pm 14.6$ \\
Male & $73.7 \%$ & $61.5 \%$ & $64.0 \%$ \\
White & $55.8 \%$ & $71.8 \%$ & $61.7 \%$ \\
BMI, kg/m ${ }^{2}$ (mean \pm SD) & $24.0 \pm 3.3$ & $29.9 \pm 8.4$ & $28.2 \pm 8.6$ \\
Disease condition & & & \\
Cellulitis/Erysipelas & $35.4 \%$ & $39.2 \%$ & $46.8 \%$ \\
Major cutaneous abscess & $31.7 \%$ & $30.3 \%$ & $30.2 \%$ \\
Wound infection & $32.9 \%$ & $30.5 \%$ & $23.0 \%$ \\
Lesion size, cm ${ }^{2}$ (mean \pm SD) & $325.7 \pm 317.8$ & $418.0 \pm 471.1$ & $466.7 \pm 479.1$ \\
Days from infection onset to treatment start & $4.3 \pm 2.1$ & $4.4 \pm 2.9$ & $4.4 \pm 2.4$ \\
Meeting SIRS criteria & $0.0 \%$ & $0.0 \%$ & $55.5 \%$ \\
Confirmed MRSA & $24.0 \%$ & $17.8 \%$ & $20.0 \%$ \\
Medical history & & & \\
Diabetes mellitus & $0.0 \%$ & $20.0 \%$ & $18.3 \%$ \\
Intravenous drug use & $23.3 \%$ & $38.7 \%$ & $20.5 \%$ \\
Hepatitis/other hepatic condition & $0.0 \%$ & $37.6 \%$ & $14.3 \%$ \\
Renal insufficiency & $0.2 \%$ & $1.6 \%$ & $2.8 \%$ \\
\hline
\end{tabular}

SIRS was defined as two or more of the following criteria: Temperature $>38^{\circ} \mathrm{C}$, pulse $>90 \mathrm{bpm}$, respiratory rate $>20$ breaths per minute, white blood cell count $>12,000 / \mathrm{mm}^{3}$, or $<4000$ or $>10 \%$ bandemia

$B M I$ body mass index, mITT modified intent-to-treat, MRSA methicillin-resistant Staphylococcus aureus, SD standard deviation, SIRS systemic inflammatory response syndrome 


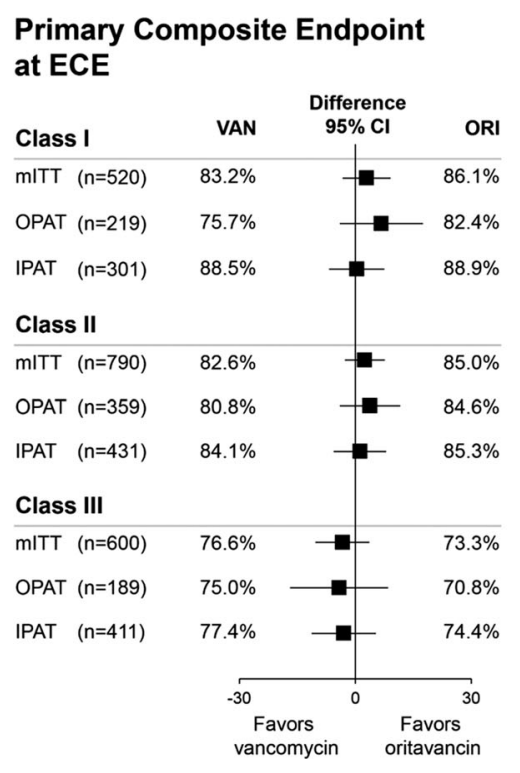

Fig. 2 Primary and secondary endpoints by classification and treatment (mITT population). CI confidence interval, $E C E$ early clinical evaluation $(48-72 \mathrm{~h}$ from treatment initiation), IPAT inpatient parenteral antibiotic therapy,

Overall $40 \%(n=767)$ of all patients in the SOLO trials were managed as outpatients. The percentages of patients treated entirely at an outpatient setting were $42.1 \%, 45.4 \%$, and $31.5 \%$ in Class I, II, and III patients, respectively (Table 2). Of the patients who were enrolled in the United States, $73 \%$ were managed in the outpatient setting and of those, $71 \%$ were Class II-III, which is similar to the overall study population. The combined efficacy for both drugs using the primary endpoint of clinical response at ECE for outpatients versus inpatients in Class I patients was $79 \%$ vs. $88.7 \%$, Class II patients $82.7 \%$ vs. $84.7 \%$, and $73 \%$ vs. $75.9 \%$ in Class III patients, respectively.

Within each Class (I-III), patients receiving oritavancin experienced similar clinical efficacy as those receiving vancomycin for the primary composite ECE outcome, lesion size reduction at ECE, and clinical cure at PTE. Treatment outcomes for oritavancin and vancomycin were also similar within each class when patients were analyzed by inpatient or outpatient management setting (Fig. 2). Response rates at ECE for patients in Class III (75.0\%) were lower than those observed with patients in Class I (84.6\%, $P<0.001)$ and Class II $(83.8 \%, P<0.001)$. However, at PTE the response rates did not differ between Class III (79.1\%) and Class I (82.3\%, $P=0.293)$ or Class II $(81.1 \%, P=0.542)$.

\section{DISCUSSION}

A clinical algorithm based on the Eron classification system that stratifies patients based on the presence of systemic symptoms of infection and comorbidities associated with poor outcomes was developed and applied to ABSSSI patients pooled from two randomized controlled clinical trials. In this post hoc analysis of the SOLO trials, Class I and II patients treated with a single-dose of oritavancin or vancomycin twice daily administered over 7-10 days had similar 
response rates if they were managed in the inpatient or outpatient settings. Results of this analysis suggest the majority of patients in Class I and II can be safely managed in the outpatient setting.

Class III patients in the SOLO trials had numerically lower responses to both drugs at ECE (oritavancin 73.3\%, vancomycin 76.6\%) when compared to Class I-II patients (82.6-86.1\%), although rates were similar between each drug and management settings. The response rates of Class III patients at PTE were approximately $80 \%$ (similar to rates in Eron Class I and II) in both vancomycin and oritavancin groups regardless of site of care. This suggests that Class III patients may have had a slower early treatment response but still achieved investigator defined clinical cure at rates similar to Class I-II patients. Of note, a smaller overall percentage (31.5\%) of Class III patients was managed in the outpatient setting. This likely represents a more guarded approach to managing patients presenting with unstable comorbidities or SIRS criteria. A lower response rate in Class III patients using an endpoint of 30-day mortality has also been identified previously [9]. Patients presenting with SIRS criteria require close monitoring and management to ensure an adequate clinical response. This management may occur as an outpatient in an observation unit or as an inpatient. Patients with a rapid clinical response may be candidates for continued management in the outpatient setting once stabilized.

One limitation to this analysis is that while the Eron/CREST treatment guidelines provide an approach to patient stratification, they have not been rigorously validated by clinical studies. The Eron classification has been criticized for being ambiguous with respect to the patient characteristics in the different severity classifications as well as being difficult to translate into real world treatment protocols
[7, 8]. However, the Eron classification incorporates several important patient factors and was shown in a retrospective analysis of Premier database that Eron Classes I-IV correlated with increasing Charlson Comorbidity Index score, proportion of inpatients, in-hospital mortality rate, length of hospital stay, cost per patient and the use of MRSA-active antibiotics [11].

\section{CONCLUSIONS}

The results demonstrate that single-dose oritavancin is an effective alternative to 7-10 days of IV vancomycin for the treatment of patients with ABSSSI within modified Eron Classes I-III. Management in the inpatient or outpatient setting was associated with comparable efficacy. Tools such as the Eron classification may be useful in the identification of patients with ABSSSI that could be managed in the outpatient setting, thereby avoiding hospitalization.

\section{ACKNOWLEDGMENTS}

Sponsorship for this study and article processing charges was funded by The Medicines Company, Parsippany, NJ, USA. All named authors meet the International Committee of Medical Journal Editors (ICMJE) criteria for authorship for this manuscript, take responsibility for the integrity of the work as a whole, and have given final approval to the version to be published. Editorial assistance in the preparation of this manuscript was provided by Mike Dudley, Greg Moeck, Mark Redell, and John Clegg of The Medicines Company.

Disclosures. Authors Daniel H. Deck, Jennifer M. Jordan, Weihong Fan, Matthew A. 
Wikler, and Katherine A. Sulham are employees of The Medicines Company. Thomas L. Holland has received payment as a consultant from The Medicines Company and Basilea. G. Ralph Corey has received payment for consultative work or speaking engagements for: Medtronic, The Medicines Company, Cerexa/Forest/ Actavis, Theravance, Pfizer, Cempra, Cubist, GlaxoSmith Kline, DRI, Nabriva, Merck, Achaogen, Melinta, Motif, Bio2 Medical, Tetraphase, Arsanis, Contrafect, Cowen Group, Paratek, Bayer, SCPharma, and Basilia.

Compliance with Ethics Guidelines. All procedures followed were in accordance with the ethical standards of the responsible committee on human experimentation (institutional and national) and with the Helsinki Declaration of 1964, as revised in 2013. Informed consent was obtained from all patients for being included in the SOLO trials. This article is based on previously conducted studies, and does not involve any new studies of human or animal subjects performed by any of the authors.

Open Access. This article is distributed under the terms of the Creative Commons Attribution-NonCommercial 4.0 International License (http://creativecommons.org/licenses/ by-nc/4.0/), which permits any noncommercial use, distribution, and reproduction in any medium, provided you give appropriate credit to the original author(s) and the source, provide a link to the Creative Commons license, and indicate if changes were made.

\section{REFERENCES}

1. Corey GR, Kabler H, Mehra P, et al. Single-dose oritavancin in the treatment of acute bacterial skin infections. N Engl J Med. 2014;370(23):2180-90.
2. Corey GR, Good S, Jiang H, Moeck G, Wikler M, Green S, Manos P, Keech R, Singh R, Heller B, Bubnova N, O'Riordan W, SOLO II Investigators. Single-dose oritavancin versus 7-10 days of vancomycin in the treatment of gram-positive acute bacterial skin and skin structure infections: the SOLO II noninferiority study. Clin Infect Dis. 2015;60(2):254-62.

3. Chambers HF. Pharmacology and the treatment of complicated skin and skin-structure infections. N Engl J Med. 2014;370(23):2238-9.

4. Stevens DL, Bisno AL, Chambers HF, et al. Practice guidelines for the diagnosis and management of skin and soft tissue infections: 2014 update by the Infectious Diseases Society of America. Clin Infect Dis. 2014;59:e10-52.

5. Eron LJ, Lipsky BA, Low DE, et al. Managing skin and soft tissue infections: expert panel recommendations on key decision points. J Antimicrob Chemother. 2003;52(Suppl 1):i3-17.

6. DHSS Northern Ireland. CREST (Clinical Resource Efficiency Support Team) guidelines on the management of cellulitis in adults. 2005. p. 1-31. http://www.acutemed.co.uk/docs/Cellulitis\%20guide lines,\%20CREST,\%2005.pdf. Accessed 27 June 2016.

7. Ki V, Rotstein C. Bacterial skin and soft tissue infections in adults: a review of their epidemiology, pathogenesis, diagnosis, treatment and site of care. Can J Infect Dis Med Microbiol. 2008;19:173-84.

8. Koerner R, Johnson AP. Changes in the classification and management of skin and soft tissue infections. J Antimicrob Chemother. 2011;66:232-4.

9. Marwick C, Broomhall J, McCowan C, et al. Severity assessment of skin and soft tissue infections: cohort study of management and outcomes for hospitalized patients. J Antimicrob Chemother. 2011;66:387-97.

10. Carratala J, Roson B, Fernandez-Sabe N, et al. Factors associated with complications and mortality in adult patients hospitalized for infectious cellulitis. Eur J Clin Microbiol Infect Dis. $2003 ; 22(3): 151-7$.

11. Sulham, K, LaPensee KT, Fan W, et al. Severity and costs of acute bacterial skin and skin structure infections by treatment setting: an application of the Eron classification to a real-world database [abstract PIN99]. In: Program of the 19th annual international meeting of the International Society for Pharmacoeconomics and Outcomes Research (Montreal, QC, Canada). Lawrenceville, New Jersey, May 31-June 4, 2014. 